\title{
Compact Representation of Knowledge Bases in ILP
}

\author{
Jan Struyf, Jan Ramon, and Hendrik Blockeel \\ Katholieke Universiteit Leuven \\ Department of Computer Science \\ Celestijnenlaan 200A, B-3001 Leuven, Belgium \\ $\{$ Jan.Struyf, Jan.Ramon, Hendrik.Blockeel\}@cs.kuleuven.ac. be
}

\begin{abstract}
Many inductive systems, including ILP systems, learn from a knowledge base that is structured around examples. In practical situations this example-centered representation can cause a lot of redundancy. For instance, when learning from episodes (e.g. from games), the knowledge base contains consecutive states of a world. Each state is usually described completely even though consecutive states may differ only slightly. Similar redundancies occur when the knowledge base stores examples that share common structures (e.g. when representing complex objects as machines or molecules). These two types of redundancies can place a heavy burden on memory resources. In this paper we propose a method for representing knowledge bases in a more efficient way. This is accomplished by building a graph that implicitly defines examples in terms of other structures. We evaluate our method in the context of learning a Go heuristic.
\end{abstract}

\section{Introduction}

Inductive logic programming is concerned with the induction of new knowledge (hypotheses) from a given knowledge base. This knowledge base is in practice often structured around the notion of examples. The aim of this paper is to find a compact representation for these examples. We start by listing some motivating applications.

1. The task in relational reinforcement learning [7] is to learn a relationship between the structural description of a state and the optimal action for that state. A typical knowledge base will contain a number of episodes, sequences of states in which each state can be reached from the previous one by taking a certain action. Storing each state independently in the knowledge base consumes a lot of memory. An alternative is to store only the initial state together with a sequence of actions. Consecutive states can be constructed by the ILP system when needed (by executing the corresponding action from the previous state) and discarded afterwards.

2. When learning to play a certain game (e.g. Chess or Go [18]), the knowledge base will consist of a number of played games (generated by an expert human 
player or by a search algorithm). Each game is represented by a number of (consecutive) board states and moves. The same compact representation as described in the previous example can be used.

3. In some applications the examples are complex objects that can be decomposed in elementary structures. For instance machines built of various parts, state descriptions of a dungeon game where one can meet several monsters and own different types of weapons, or molecules that contain several functional groups. Representing examples independently in these cases will cause redundancy. This redundancy can be avoided by representing examples implicitly as a combination of their elementary structures.

4. Many tasks consist of classifying an individual element in a sequence based on its local context. Consider for instance protein secondary structure prediction [17], part of speech tagging [5] or user modeling [14]. Consecutive examples in this kind of knowledge bases are similar because the neighborhood of adjacent positions is similar. A compact representation is possible by defining a window for each example instead of explicitly storing the neighborhood.

As can be seen from the examples above, we need a formalism to represent individual examples in terms of previous examples (i.e. by using actions or, more generally, difference information) and in terms of elementary structures. We will introduce such a formalism in this paper.

Note that it is also possible to represent the set of examples more compactly in an ILP system by defining background knowledge $B$. For example, one could define different machine parts in $B$ or one could represent state information implicitly using predicates defined in $B$. However, using this approach $B$ must be redefined for each specific application. In this paper we propose a more general approach.

Using background knowledge for defining examples has two other disadvantages. (1) The ILP system must be able to load $B$ in main memory. If we use $B$ to store parts of the example descriptions (e.g. machine parts), then this puts a bound on the number of examples that the ILP system can use. (2) When using implicit state descriptions, the time for accessing a single example (state $s_{i}$ ) will depend on the number of previous states (we have to iterate over all previous states to obtain the definition of $s_{i}$ ). This means that the computational complexity of querying all examples (in random order) will be at least quadratic in the number of examples.

Scalability to large knowledge bases is obtained in practical ILP systems either (1) by connecting the ILP system to an external database management system (DBMS) $[1,15,13]$ or (2) by using independent examples that can be loaded one by one, i.e. the Learning from Interpretations setting [3] in which each example is defined as a small relational database (typically implemented as a set of facts, i.e. an interpretation). The benefit of (2) over (1) is that (1) must rely for efficiency on the caching mechanism provided by the DBMS which lacks information about the specific way in which the ILP system will access the examples. In (2) this information is made explicit by combining all data relevant to a single example in an interpretation. This makes retrieving a single example 
very efficient. The drawback of (2) is that examples have to be independent which means that compact representation (by defining examples in terms of other structures) is impossible. The framework presented in this paper extends the Learning from Interpretations setting by allowing dependent examples without sacrificing scalability.

The remainder of the paper is structured as follows. In Section 2 we introduce a formalism (the knowledge base graph or $K B G$ ) which enables us to represent examples in terms of other structures. In Section 3 we illustrate how the $K B G$ can be described in Prolog. We discuss how an ILP algorithm can query the examples defined by the $K B G$ efficiently in Section 4 . Section 5 presents preliminary experiments evaluating our method in the context of learning a Go heuristic. In Section 6 we state the conclusions.

\section{Knowledge Base Graph}

In this section we propose the knowledge base graph or $K B G$ as a formalism for representing examples in terms of other structures. We start by defining the $K B G$ formally. After that we show that the $K B G$ can be used to compactly represent each type of knowledge base mentioned in the introduction.

\subsection{Hypergraphs}

As the $K B G$ is in fact a directed hypergraph, we would like to first briefly review directed hypergraphs. Directed hypergraphs are a generalization of normal graphs because they contain hyperedges which are able to express many-to-one relations (normal graph edges only relate two nodes).

Definition 1. A directed hypergraph $\mathcal{H}$ is a pair $(N, \mathcal{E})$ where $N$ is a set of nodes and $\mathcal{E}$ is a set of hyperedges. Each hyperedge $\epsilon$ is a tuple $\left(n_{1}, \ldots, n_{k}, n\right)$ from a set $\operatorname{source}(\epsilon)=\left\{n_{1}, \ldots, n_{k}\right\} \subseteq N$ (source set) to a single node $n \in N$ (target node).

Hypergraphs (See [11] for a discussion) are widely used in operations research and computer science (e.g. to model functional dependencies in relational databases, to model assembly plans and to represent Horn clauses, implications and and-or graphs). Hypergraphs are also used in knowledge representation [8] and [19] uses the hypergraph representation to parallelize a Knowledge Discovery System that embeds an ILP engine.

\section{$2.2 \quad$ Structure}

The $K B G$ is a directed hypergraph with two sets of nodes: explicit nodes $N_{E}$ and implicit nodes $N_{I}$. The explicit nodes store information from some domain $D$. We refer to the elements of $D$ as "elementary structures" or "objects". In practice, elementary structures can be sets of facts, partial logic programs (sequences of clauses), terms [10], or application specific objects (Section 5). 


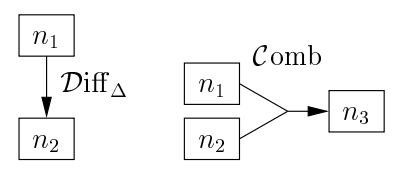

Fig. 1. Graphical representation of $\mathcal{D}$ iff and $\mathcal{C}$ omb hyperedges.

The implicit nodes do not store objects. However it is possible to compute an object associated with each implicit node. Computing this object is called explicitating the node. More formally, we define a function expl (explicitate) that maps each node to an object $o \in D$. For explicit nodes expl retrieves the object stored in the node. For implicit nodes, expl is defined (implicitly) by the hyperedges of the $K B G$.

Definition 2. A difference hyperedge Diff is a tuple $\mathcal{D i f f}_{\Delta}\left(n_{1}, n_{2}\right)$ with $n_{1} \in$ $N_{E} \cup N_{I}$ and $n_{2} \in N_{I}$. We have $\mathcal{D i f f}_{\Delta}\left(n_{1}, n_{2}\right)$ iff expl $\left(n_{2}\right)$ can be derived from $\operatorname{expl}\left(n_{1}\right)$ by applying difference information $\Delta$.

Each difference hyperedge $\mathcal{D}$ iff stores some information $\Delta$ that describes how $\operatorname{expl}\left(n_{2}\right)$ can be derived from expl $\left(n_{1}\right)$. In the context of games for example, $\Delta$ could state that a particular piece is placed on the board. We represent a difference hyperedge graphically as shown in Figure 1.

Definition 3. A combine hyperedge $\mathcal{C}$ omb is a tuple $\mathcal{C}$ omb $\left(n_{1}, n_{2}, n_{3}\right)$ with $n_{1}, n_{2} \in N_{E} \cup N_{I}$ and $n_{3} \in N_{I}$. We have $\mathcal{C} \operatorname{omb}\left(n_{1}, n_{2}, n_{3}\right)$ iff $\operatorname{expl}\left(n_{3}\right)$ can be obtained by combining expl( $\left.n_{1}\right)$ and expl $\left(n_{2}\right)$.

The effect of combining $\operatorname{expl}\left(n_{1}\right)$ and $\operatorname{expl}\left(n_{2}\right)$ has to be defined for each specific choice of $D$. For example, if the objects are sets of facts, then the natural implementation for combining objects would be to take the union of these sets. We represent $\mathcal{C}$ omb hyperedges graphically as shown in Figure 1.

In this paper we consider only $\mathcal{D}$ iff and $\mathcal{C}$ omb hyperedges. However, the $K B G$ can be extended by defining more types of hyperedges. Note that $\mathcal{C}$ omb hyperedges can also be extended to hyperedges that combine more than two nodes.

Definition 4. The example nodes $E_{N} \subseteq N_{E} \cup N_{I}$ are the nodes of interest to the ILP system. The examples $E_{o}$ are the objects associated with the example nodes $\left(E_{o}=\left\{\operatorname{expl}(n) \mid n \in E_{N}\right\}\right)$.

\section{$2.3 \quad$ Examples}

In this section we show how some example knowledge bases can be represented using a $K B G$.

1. The Learning from Interpretations setting corresponds to the trivial form of the $K B G$ when $N_{E}=E_{N}, N_{I}=\emptyset, \mathcal{E}=\emptyset$. In this case all examples are represented explicitly (i.e. there is no sharing). 
2. Background knowledge $B$ can be viewed as a node $n_{B}$ that is combined with example descriptions $e d_{i} \in E D$ (e.g. explicit nodes that store sets of facts) to form example nodes $e_{i} \in E_{N}\left(N_{E}=\left\{n_{B}\right\} \cup E D, N_{I}=E_{N}\right.$, $\mathcal{E}=\left\{\mathcal{C}\right.$ omb $\left.\left.\left(e d_{i}, n_{B}, e_{i}\right) \mid e d_{i} \in E D, e_{i} \in E_{N}\right\}\right)$.

3. When learning from episodes (e.g. in game play), we have one initial state for each episode $i$. Initial states $s_{i, 0}$ are represented in the $K B G$ as explicit nodes $\left(\operatorname{expl}\left(n_{i, 0}\right)=s_{i, 0}, n_{i, 0} \in N_{E}\right)$. Consecutive states $s_{i, j}$ are not stored explicitly and hence correspond to implicit nodes $\left(\operatorname{expl}\left(n_{i, j}\right)=s_{i, j}, n_{i, j} \in\right.$ $N_{I}$ ). Diff hyperedges are used to describe the difference between $s_{i, j}$ and $s_{i, j+1}$. In this setting, the $K B G$ is a set of sequences.

4. Sometimes a state can have several successors. This is useful for representing a number of possible actions in a state. In the context of games, a game subtree can be represented in this way. This is useful for learning from alphabeta search trees (Section 5) or from games that allow variations. In this setting, the $K B G$ is a set of trees.

5. It is also possible that some states can be reached with different sequences of moves/actions. If this happens, then the $K B G$ will be a directed acyclic graph.

6. If examples share common substructures then we can use $\mathcal{C}$ omb hyperedges. For the machines example it seems natural to have one (explicit) node for each part. Each example corresponds to an implicit node (a machine) that can be obtained by combining the different parts. Knowledge bases that store molecules or sequences can be represented in a similar way.

7. Comb hyperedges can also be used in combination with $\mathcal{D}$ iff hyperedges. Consider again the learning from game play example. Suppose that the examples for the ILP algorithm are the legal moves in each state. In this case the example nodes are implicit move-nodes that can be obtained by combining explicit move information (e.g. coordinates and value of the move) with the corresponding implicit state-node.

Note that when all possible actions are computable from a state (as is the case with most board games), the hyperedges and the implicit nodes of the $K B G$ could be computed lazily. In that case the set of all possible games can be represented compactly by defining the initial state and a function mapping states to possible actions. The difference between a finite database of games and a world in which the learner can experiment becomes entirely transparent, offering an elegant method for active learning and random sampling.

\section{Representing the $K B G$ in Prolog}

In this section we illustrate how the $K B G$ can be described in Prolog for the case where each object is a partial logic program (PLP). We will use Go [18] as example application. Go, a game popular in Asia is an abstract two-person complete-information deterministic board game (like Chess and Draughts). 


\subsection{Defining Explicit Nodes}

Each explicit node stores a PLP (in our example the definition of a Go board state) and can be defined by placing the PLP between the keywords begin_explicit_node(n) and end_explicit_node(n) (Explicit nodes are represented graphically with a double rectangle).

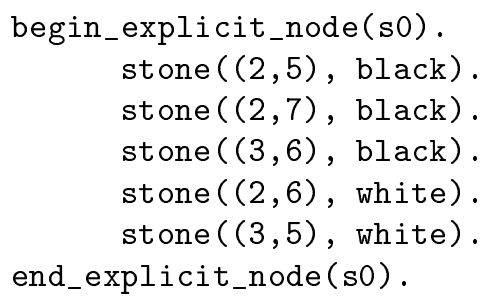

\subsection{Defining $\mathcal{D}$ iff hyperedges}

We define $\mathcal{D}$ iff hyperedges using diff_edge/3. The first argument is the source node, the second argument the target node and the last argument specifies $\Delta$. We can distinguish two abstraction levels for defining $\Delta$. The first abstraction level is directly describing the difference between objects. In this case $\Delta$ specifies transformations on the PLP.
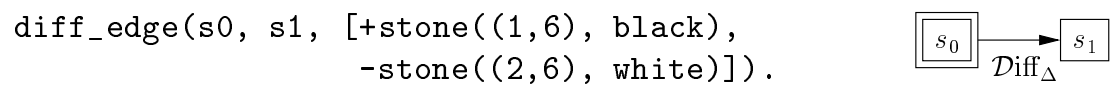

In the example above $\Delta$ describes which clauses have to be added to $(+)$ or removed from (-) the PLP. This way of representing $\mathcal{D}$ iff hyperedges is very similar to the way operators are defined for a STRIPS [9] planner.

The second abstraction level is defining $\Delta$ using actions. In the Go example, this corresponds to storing moves instead of explicit object differences.

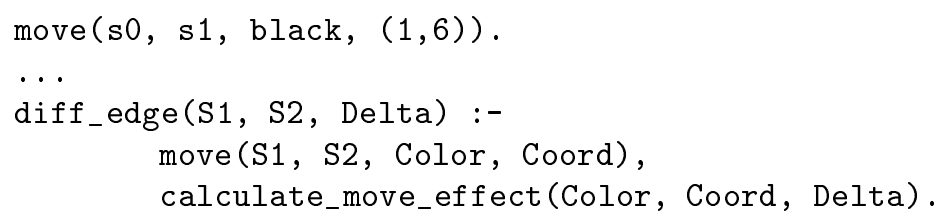

Note that calculate_move_effect/3 will in most cases depend on the current state (i.e. the PLP associated with S1). This means that the knowledge base has to explicitate $\mathrm{S} 1$ before using the diff_edge/3 definition.

\subsection{Defining $\mathcal{C}$ omb hyperedges}

$\mathcal{C}$ omb hyperedges can be defined using comb_edge/3. The semantics of a $\mathcal{C}$ omb$\left(n_{1}, n_{2}, n_{3}\right)$ hyperedge are that the definitions of the PLP's associated with $n_{1}$ and $n_{2}$ are concatenated. In the Go example below, a move-node is defined by combining move information (e.g. the player who made the move, the move's coordinates and evaluation score) with a state-node. 


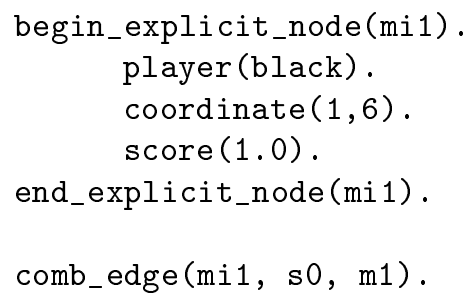

\subsection{Defining Examples}

The example nodes (in this case the moves) are a subset of the nodes of the $K B G$ (Definition 4). We define this subset using example/1.

example (m1).

example (m2).

..

\section{Traversing the $K B G$}

In order to be practically useful, a $K B G$-based knowledge base should satisfy two key properties. The first one is transparency: the interface for interacting with the knowledge base should be the same as when all examples are represented explicitly. Transparency implies that existing ILP systems can be adapted easily to use a $K B G$-based knowledge base. The second property is computational efficiency: some loss of efficiency when using the $K B G$ may be unavoidable, but it should remain limited (e.g. querying all examples should be $\mathcal{O}(N)$ with $N$ the number of nodes in the $K B G$ ).

\subsection{ILP Algorithm Interface}

We call the interface component between the ILP algorithm and the $K B G$ the example iterator. The task of the example iterator is to traverse the $K B G$ and explicitate the example nodes that the ILP algorithm needs to query. Most ILP algorithms consider different subsets $S$ of the available examples without imposing an order on the elements of $S$. This means that the example iterator can choose an order for iterating over $S$. Consider for example the $K B G$ shown in Figure 2. The example iterator starts with the explicit node $e_{1}$. After that, it can move to $e_{2}$, explicitate $e_{2}$ by applying $\Delta 2$ and proceed with $e_{3}$. This means that the sequence $e_{1}, e_{2}, e_{3}$ can be iterated efficiently. A sequence like $e_{3}, e_{2}, e_{1}$ is less efficient because it is impossible to explicitate $e_{3}$ without constructing $\operatorname{expl}\left(e_{2}\right)$ first. In Section 4.2 we will introduce the notion of this order in a more formal way. We conclude this section with some remarks on how different ILP algorithms query examples.

1. Some ILP algorithms build a model by considering different subsets of the available examples (e.g. PROGOL [16], TiLde [2]). Other algorithms perform 


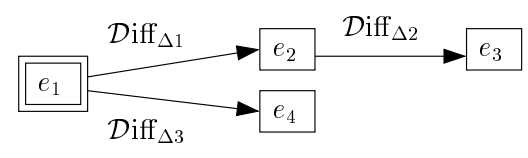

Fig. 2. An example $K B G$ structure.

different scans over the entire knowledge base (e.g. WARMr [6]). Algorithms that perform full scans will execute more efficiently in combination with the $K B G$. Consider for example Figure 2 and suppose that the algorithm partitions the examples in $S_{1}=\left\{e_{1}, e_{3}\right\}, S_{2}=\left\{e_{2}, e_{4}\right\}$. In this case expl $\left(e_{2}\right)$ has to be constructed twice: once as intermediate result for $\left\{e_{1}, e_{3}\right\}$ and once for $\left\{e_{2}, e_{4}\right\}$. If the algorithm performs scans over the entire set $\left\{e_{1}, e_{2}, e_{3}, e_{4}\right\}$ then $\operatorname{expl}\left(e_{2}\right)$ has to be constructed only once.

2. Most ILP algorithms evaluate a set of queries $Q$ on a set of examples $S$. This can be implemented in two ways: execute each query on all examples (queries outer loop) or execute all queries on each example (examples outer loop). When used in combination with a $K B G$ examples outer loop algorithms are more efficient. This is because the examples have to be constructed only once instead of once for each query. Note that full scan algorithms typically implement the examples outer loop setting.

3. Incremental ILP algorithms can use an infinite $K B G$. They access each example only once. This is also true for reinforcement learning [7]. Most other algorithms need a finite set of examples or work with different finite batches of examples.

\subsection{A Planning Problem}

The task of the example iterator is to construct each example $e \in S$ so that the ILP algorithm can query it. The example iterator must traverse the $K B G$ in a certain order to accomplish this (consider again Figure 2). In the most general case, finding this order corresponds to solving a planning problem. Before we define this planning problem, we introduce the notion of active nodes.

A node $n$ for which $\operatorname{expl}(n)$ is in the main memory of the example iterator is called an active node (cached node). The example iterator can activate a node depending on its type.

1. An explicit node $n$ can always be activated (by retrieving the object stored in $n$, i.e. loading $\operatorname{expl}(n)$ from $\left.\operatorname{disk}^{1}\right)$.

2. An implicit node $n$ can only be activated if there exists a hyperedge $\epsilon$ with $n$ as target node and for which all nodes in the source set are active. Activating the node corresponds to constructing $\operatorname{expl}(n)$ as defined by $\epsilon$.

The reverse action, deactivating $n$, corresponds to removing $\operatorname{expl}(n)$ from the main memory.

\footnotetext{
${ }^{1}$ This functionality could be provided by an object oriented DBMS.
} 


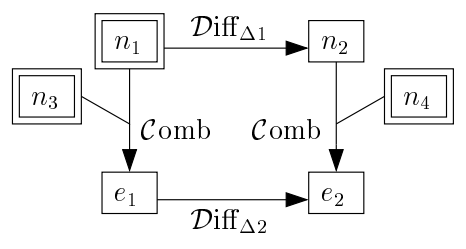

Fig. 3. A Planning Problem.

While deactivating a node can be seen as effortless, activating a node $n$ involves a certain cost. For explicit nodes this is the cost of loading expl(n) from disk and for implicit nodes it is the cost of constructing expl $(n)$. The example iterator should minimize this cost while performing its task. The number of active nodes at any given time should also be limited. This is because the main memory of the example iterator can only store a finite number of objects. More formally, it must hold that $\left|N_{A}\right| \leq \max$ with $N_{A}$ the set of active nodes and max the maximum number of objects that can be stored in memory. Maintaining memory usage can be done by deactivating nodes that are no longer necessary.

We now define the planning problem faced by the example iterator.

Definition 5. (Planning problem faced by the example iterator) Given a set of active nodes $N_{A}=N_{A}(0)$ and a set of examples $S$ that the ILP algorithm needs to query, find a sequence of actions $a_{i}$ (activating or deactivating a node $n_{i}$ ) such that:

1. (Objective) $\sum_{a_{i}} \operatorname{cost}\left(a_{i}\right)$ is minimal.

2. (Memory constraint) $\forall i,\left|N_{A}(i)\right| \leq \max$.

3. (ILP algorithm constraint) $\forall e \in S, \exists i$ such that $e \in N_{A}(i)$.

with cost $\left(a_{i}\right)$ the cost of performing $a_{i}$ as defined above and $N_{A}(i)$ the set of active nodes after performing $a_{i}$.

Example 1. Consider the $K B G$ shown in Figure 3. Suppose that the ILP algorithm needs to query the examples $S=\left\{e_{1}, e_{2}\right\}$ and that $\max =3$. The sequence activate $\left(n_{1}\right)$, activate $\left(n_{3}\right)$, activate $\left(e_{1}\right)$, deactivate $\left(n_{3}\right)$, activate $\left.\left(e_{2}\right)\right\}$ is a plan that satisfies both the memory and the ILP algorithm constraint. Whether or not this plan is optimal depends on the costs associated with the actions. For example if $\mathcal{D}$ iff $_{\Delta 2}$ implies a high cost, then $\left\{\right.$ activate $\left(n_{1}\right)$, activate $\left(n_{3}\right)$, activate $\left(e_{1}\right)$, deactivate $\left(n_{3}\right)$, activate $\left(n_{2}\right)$, deactivate $\left(n_{1}\right)$,deactivate $\left.\left(e_{2}\right)\right\}$ may be a cheaper solution.

The planning problem faced by the example iterator is in general NP-hard. This can be seen by considering a special instance that is equivalent with the Traveling Salesman Problem (TSP). Suppose that the $K B G$ has one explicit node, a number of implicit nodes and that all hyperedges are $\mathcal{D}$ iff's. All nodes are examples for the ILP algorithm. If $\max =2$ then traversing $\mathcal{D}$ iff $\Delta\left(n_{1}, n_{2}\right)$ implies first activating $n_{2}$ and afterwards deactivating $n_{1}$. In this case the planning problem is reduced to that of finding a minimal cost path through the $K B G$ while visiting all nodes once. This is a variant of TSP. 


\subsection{An Efficient Example Iterator}

In practice we need an algorithm that scales well in the number of nodes of the $K B G$. For some specific structures of the $K B G$ it is possible to solve the planning problem efficiently. For example, if the $K B G$ is a set of sequences then the planning problem becomes trivial. For other structures it is more difficult. We propose an efficient algorithm that approximates the planning problem for a subset of all possible $K B G$ structures which we call restricted $K B G$ structures $(R K B G)$.

Definition 6. (Restricted $K B G$ structure). Let $T \in \mathbb{R}^{+}$be a constant. If it holds for a certain KBG structure and for an increasing number of nodes $N$ that:

1. Activating an explicit node can be done in time $\leq T$.

2. Activating an implicit node $n$ can be done in time $\leq T$ if $\exists \epsilon \in \mathcal{E}$ that defines $n$ for which $\operatorname{source}(\epsilon) \subseteq N_{A}$.

3. For each $\mathcal{C o m b}\left(n_{1}, n_{2}, n_{3}\right)$ hyperedge either expl $\left(n_{1}\right)$ or expl $\left(n_{2}\right)$ can be computed (using the algorithm shown in Figure 4) in time $\leq T$. We call the corresponding node (either $n_{1}$ or $n_{2}$ ) the efficient node of the $\mathcal{C}$ omb hyperedge.

then we call this $K B G$ structure a restricted $K B G$ structure.

Definition 7. (Forest). A KBG is called a forest if each node $n$ is the target of at most one hyperedge.

A $K B G$ structure that satisfies Definition 6 can be iterated in linear $\mathcal{O}(N)$ time, with $N$ the number of nodes. One can implement such a linear time example iterator as follows.

1. Compute the spanning forest (Definition 7 ) of the $K B G$. This can be done in $\mathcal{O}(N)$. The $K B G$ of Figure 3 can for example be transformed into a forest by removing $\mathcal{D i f f}_{\Delta 2}$. A better approximation of the planning problem is obtained by calculating the minimal spanning forest. This can be done using an adapted version of Prim-Jarník's [12] algorithm in $\mathcal{O}(E \log N)$, with $E$ the number of hyperedges and $N$ the number of nodes of the $K B G$. Computing the spanning forest should be done only once when the knowledge base is initialized.

2. Find the root set $R$ of the spanning forest. The root set is the set of all explicit nodes that are not ancestors of efficient nodes (See Definition 6). The root set of the $K B G$ shown in Figure 3 is $R=\left\{n_{1}\right\}$. Explicit nodes $n_{3}$ and $n_{4}$ are not in the root set because they are efficient nodes.

3. Repeat steps 4-6 for each root $r \in R$.

4. Activate $r$ and traverse the tree with root $r$ depth-first. Traversing a hyperedge means activating the corresponding child node (See steps 5 and 6). A node can be deactivated after all of its children have been visited.

5. Traversing a $\operatorname{Diff}_{\Delta}\left(n_{1}, n_{2}\right)$ hyperedge is trivial: construct expl $\left(n_{2}\right)$ by applying $\Delta$ to $\operatorname{expl}\left(n_{1}\right)$. 


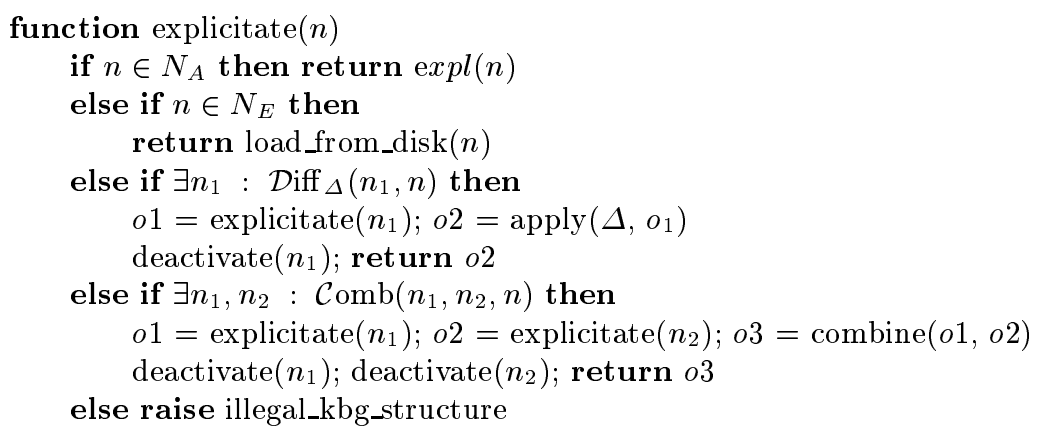

Fig. 4. An algorithm for constructing $\operatorname{expl}(n)$.

6. Traversing a $\mathcal{C} \operatorname{Omb}\left(n_{1}, n_{2}, n_{3}\right)$ hyperedge is more difficult. Suppose that $n_{2}$ is its efficient node. Before we can activate $n_{3}$, we must compute $\operatorname{expl}\left(n_{2}\right)$. Because of Definition 6, we know that this can be done efficiently (using the algorithm shown in Figure 4). After $n_{2}$ is activated we can construct expl $\left(n_{3}\right)$ by combining expl( $\left.n_{1}\right)$ and $\operatorname{expl}\left(n_{2}\right)$. Afterwards, $n_{2}$ can be deactivated.

Example 2. Consider again the $K B G$ shown in Figure 3. Removing $\mathcal{D i f f}_{\Delta 2}$ results in the root set $R=\left\{n_{1}\right\}$. We now illustrate steps 4-6. First, we activate $n_{1}$ by loading it from disk. Root $n_{1}$ has two children: $n_{2}$ and $e_{1}$. Suppose that the algorithm starts by activating $n_{2}$. The next step is traversing the $\mathcal{C}$ omb hyperedge leading to $e_{2}$. This is trivial because $n_{4}$ is an explicit node. Node $n_{2}$ can be deactivated because it has only one child. $e_{2}$ can also be deactivated (after passing expl $\left(e_{2}\right)$ to the ILP algorithm). Root $n_{1}$ has one child left: $e_{1}$. Activating this child is analogous to activating $e_{2}$. The last step of the algorithm is deactivating $n_{1}$ after all its children have been processed.

We end this section with some remarks about our algorithm.

1. The number of nodes that can be active at a given time is bounded by the maximal tree depth $d$. In most practical cases $d$ is small compared to the number of nodes. Note that we should only keep internal nodes active that have more than one successor: for sequences, the algorithm deactivates each node immediately. If the number of active nodes grows too large (the objects do not fit in memory), then the algorithm should deactivate some nodes. This implies that some objects have to be reconstructed which can increase the computational complexity of the algorithm.

2. If the efficient nodes of two $\mathcal{C}$ omb hyperedges share some ancestors, then these ancestors are constructed several times. If enough memory is available, it may be better to cache these ancestors (i.e. keep them active). It can also be useful to keep explicit nodes active in order to avoid loading them multiple times.

3. Sometimes the hyperedges of the $K B G$ can be traversed in the opposite direction. For a $\mathcal{D}$ iff hyperedge this means undoing $\Delta$. In this case it is not 
necessary to keep nodes with more than one child active until all children are processed. This method can be combined with destructive updating the objects. For some applications it is more efficient to change an existing object than to construct a new object. For example in Go it is much more efficient to put one stone on an existing board than constructing a new board with the extra stone. Destructive updates can only be done if the source node may be deactivated after traversing a given hyperedge.

4. If the ILP algorithm needs to query small subsets $S$ then it is possible that some parts of the $K B G$ are irrelevant. Consider for example Figure 3 and suppose that $S=\left\{e_{1}\right\}$. This implies that $e_{2}, n_{4}$ and $n_{2}$ are not needed anymore. An obvious optimization to the proposed algorithm is to start with a step that marks all nodes (indirectly) needed by the ILP algorithm (in the example $e_{1}, n_{3}$ and $n_{1}$ ). In step 4 it is then enough to consider trees with marked roots. When traversing these trees, unmarked children do not need to be processed.

\section{Experimental evaluation}

In order to evaluate our method we did some preliminary experiments on an application in the context of Go [18]. Unlike other two-person complete-information deterministic games such as Chess and Draughts, brute force search is not sufficient to let computers play Go at a level comparable to human players. Therefore, research is necessary on more intelligent methods. One of such is to learn heuristics in order to solve local problems more efficiently. In [18] we applied the TILDE $[2,4]$ system (a first order decision tree induction system) to a set of local problems to learn a heuristic that can be used in an alpha-beta search to order moves and hence improve pruning of bad moves. In [18] we only predicted the first move that should be done in a problem. Further research now focuses on integrating the TILDE system and the search algorithm to learn better heuristics. The search algorithm first solves a problem, and all the nodes it visits are logged. Afterwards, heuristics are learned to optimize the search process.

From the log generated by the search algorithm we can construct a $K B G$ as shown in Figure 5. The backbone of this $K B G$ is formed by the states visited by the alpha-beta search (state-nodes $s_{i}$ connected with $\mathcal{D}$ iff hyperedges).

The search algorithm uses an iterative deepening method. In each iteration a number of states are expanded further, i.e. more nodes of the subtree under that node are investigated. Hence each state-node of the tree is expanded one or more times. A node_expand node in the $K B G$ can be constructed by combining a state-node with a node_expand_info node. This node_expand_info node contains information specific to a particular visit of the algorithm to the corresponding state-node, such as the amount of time spent and the depth to which this node was expanded. In each visit a set of candidate moves is investigated. This introduces a third kind of implicit nodes in the $K B G$ : the move-nodes. A move-node is defined by combining a node_expand node and a move_info node that contains the coordinates of the move, the value of the move that was calculated during 


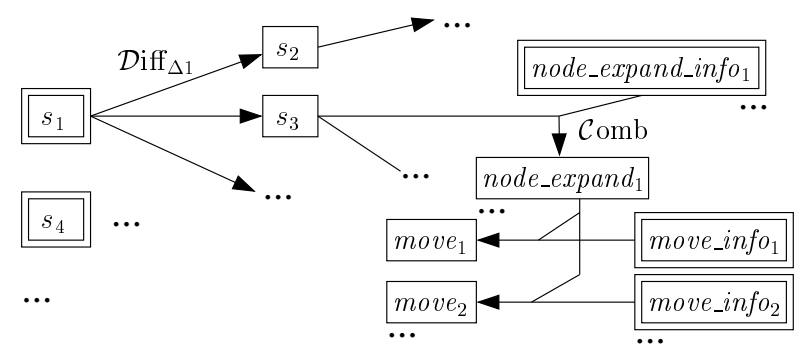

Fig. 5. The $K B G$ for the Go application.

this particular node expansion, a measure for the difficulty to find an accurate estimate of the value of the move, etc.

We have implemented a $K B G$ in the knowledge base of the ILP system ACE [4] (which includes TILDE). For the Go application, the example iterator does not cache objects at internal nodes of the $K B G$ trees. Instead it uses destructive updating of the Go states as discussed in Section 4.3. The example iterator loads all objects associated with explicit nodes in main memory during initialization. Our current implementation does not have a caching strategy for on-demand loading. It does not include support for manipulating PLP's either. Instead, we use an application specific library ${ }^{2}$ written in $\mathrm{C}++$ designed to efficiently represent and manipulate Go states.

Table 1 shows a comparison between the compact (i.e. using the $K B G$ described above) and full (all board states are represented explicitly ${ }^{3}$ ) representation of the Go knowledge base. Each row corresponds to a single experiment for which the knowledge base was obtained by changing parameters (max number of nodes to visit, minimal depth of the search that is logged) of the search algorithm. The first column contains the number of moves (i.e. examples). The other columns contain the sizes of the knowledge base on disk and the runtime of the TILDE system. We measured runtimes using the Query packs [4] and the queries outer loop setting of TILDE. Query packs is an optimized version of examples outer loop (Section 4.1).

When using the Query pack ${ }^{4}$ mechanism, the differences in runtime between the experiments on the compact and full representation are minimal (even difficult to measure). We also tried the less efficient query outer loop setting (Section 4.1), where the ILP system iterates for each candidate query over all examples. As this approach needs more computational effort of the example iterator, the experiments on the compactly represented knowledge base are slightly slower. In the last row of the table, larger overhead occurs (6\% resp. 10\%). This overhead

\footnotetext{
${ }^{2}$ Available for academic purposes upon request.

${ }^{3}$ If more than one move is explored for a given board state, then this state is not replicated for each move.

${ }^{4}$ The Query pack experiments run efficient because the Query packs have a long root and a high branching factor in this application. Query packs are also pre-compiled.
} 


\begin{tabular}{|c|c|c|c|c|c|c|c|c|c|}
\hline \multirow[t]{2}{*}{ \# moves } & \multicolumn{3}{|l|}{ Size } & \multicolumn{3}{|c|}{ Query packs } & \multicolumn{3}{|c|}{ Queries outer loop } \\
\hline & Full & Comp & & Full & Comp & & Full & Comp & \\
\hline 1269 & 634 & 153 & $4.1 \times$ & 11.9 & 12.1 & $-2 \%$ & 864 & 840 & $3 \%$ \\
\hline 2795 & 1580 & 369 & $4.3 \times$ & 17.9 & 17.5 & $2 \%$ & 1270 & 1250 & $2 \%$ \\
\hline 5669 & 4047 & 845 & $4.8 \times$ & 52.9 & 52.7 & $0 \%$ & 5180 & 5110 & $1 \%$ \\
\hline 9384 & 9668 & 1919 & $5.0 \times$ & 28.2 & 28.1 & $0 \%$ & 793 & 788 & $1 \%$ \\
\hline 14549 & 21776 & 4173 & $5.2 \times$ & 59.6 & 59.6 & $0 \%$ & 1200 & 1190 & $1 \%$ \\
\hline 172411 & 194098 & 37925 & $5.1 \times$ & 288.0 & 272.0 & $6 \%$ & 3840 & 3490 & $10 \%$ \\
\hline
\end{tabular}

Table 1. Comparison between the compact and full representation of the Go knowledge base. We report the size (in $\mathrm{Kb}$ ) of the knowledge base on disk and the runtime (in seconds) of the TILDE system (Query packs [4] and queries outer loop).

is introduced by the sampling method of TILDE (TILDE uses samples of 1000 moves at each node of the decision tree). If TILDE queries only a small subset of states in the $K B G$ then the overhead will increase because intermediate states have to be constructed in the compact representation without being used.

On the other hand one can see that the compactly represented knowledge bases are a factor of magnitude smaller than the full knowledge bases. The reduction is about $4 \times$ for small experiments and increases to about $5 \times$ for larger ones. The factor increases because the $K B G$ 's of larger experiments have more implicit state-nodes (i.e. nodes of the search tree) compared to the number of stored initial states.

Our experiments demonstrate that it is possible to obtain a significant reduction in memory consumption at virtually no extra computational cost by using a $K B G$-based knowledge base for Go. For other applications, where states are more complex (in Go, board states are represented very efficiently using only 2 bits for each field), we expect larger reductions in memory consumption. Because we use an application specific library for manipulating Go states $\mathcal{D}$ iff and $\mathcal{C}$ omb operations are very efficient. If we use PLP's we can expect that these operations will be more complex, yielding a larger overhead. For large knowledge bases, the time necessary for loading objects may be larger than the time necessary for constructing them. If this is the case then it is possible that the runtime actually decreases when using a $K B G .^{5}$

\section{Conclusions}

In many applications, knowledge bases for ILP algorithms store redundant information. Typical examples are learning from episodes where consecutive states of a world have to be stored, and learning from objects composed from elementary structures. In this paper we introduced a formalism, the knowledge base graph $(K B G)$, which is able to represent examples implicitly in terms of other

\footnotetext{
${ }^{5}$ The time for loading the knowledge base was not included in the reported runtimes.
} 
structures. Using this formalism, it is possible to remove most of the redundancy from the knowledge base.

Most ILP systems can be adapted easily to work with a $K B G$. In order to accomplish this we introduced an interface between the ILP system and the knowledge base: the example iterator. The example iterator has to iterate the examples stored in the $K B G$ in an optimal order. This corresponds to an NPhard planning problem for which we propose an efficient approximation that can iterate the examples in linear time (for the case of a restricted $K B G$ ).

We evaluated our method in the context of learning a Go heuristic. For this application it is possible to obtain a significant reduction in memory consumption (up to $5 \times$ ) at virtually no extra computational cost $(\leq 10 \%)$. We also briefly discussed how we expect these results to generalise to other applications.

Further work includes more experiments with other knowledge bases. The first thing we would like to investigate is knowledge bases that store partial logic programs (PLP's). In order to manipulate PLP's efficiently it would be good to have special support from the Prolog engine. Furthermore, we would like to look at knowledge bases that are lazily sampled from the whole example space. For some applications (e.g. knowledge bases storing molecules) it is less obvious how to decompose structured objects. For these types of applications, it would be interesting to have a system that could separate common substructures automatically. Such a system is perhaps more generally applicable for optimizing the compilation of a knowledge base.

\section{Acknowledgments}

Jan Struyf is a research assistant, Hendrik Blockeel a post-doctoral fellow of the Fund for Scientific Research (FWO) of Flanders. Jan Ramon is supported by the Flemish Institute for the Promotion of Science and Technological Research in Industry (IWT). The presentation of this paper at ILP'02 is supported by ILPnet2 (http://www.cs.bris.ac.uk/ ${ }^{\sim}$ ILPnet2/).

\section{References}

1. H. Blockeel and L. De Raedt. Relational knowledge discovery in databases. In Proceedings of the Sixth International Workshop on Inductive Logic Programming, volume 1314 of Lecture Notes in Artificial Intelligence, pages 199-212. SpringerVerlag, 1996.

2. H. Blockeel and L. De Raedt. Top-down induction of first order logical decision trees. Artificial Intelligence, 101(1-2):285-297, June 1998.

3. H. Blockeel, L. De Raedt, N. Jacobs, and B. Demoen. Scaling up inductive logic programming by learning from interpretations. Data Mining and Knowledge Discovery, 3(1):59-93, 1999.

4. H. Blockeel, L. Dehaspe, B. Demoen, G. Janssens, J. Ramon, and H. Vandecasteele. Improving the efficiency of inductive logic programming through the use of query packs. Journal of Artificial Intelligence Research, 16:135-166, 2002. 
5. J. Cussens. Part-of-speech tagging using progol. In Proceedings of the Seventh International Workshop on Inductive Logic Programming, Lecture Notes in Artificial Intelligence, pages 93-108. Springer-Verlag, 1997.

6. L. Dehaspe and H. Toivonen. Discovery of frequent datalog patterns. Data Mining and Knowledge Discovery, 3(1):7-36, 1999.

7. S. Džeroski, L. De Raedt, and K. Driessens. Relational reinforcement learning. Machine Learning, 43:7-52, 2001.

8. A. Fall and G. W. Mineau. Knowledge retrieval, use and storage for efficiency. Computational Intelligence, 15:1-10, 1999.

9. R.E. Fikes and N.J. Nilsson. STRIPS: a new approach to the application of theorem proving to problem solving. Artificial Intelligence, 2:189 - 208, 1971.

10. P.A. Flach. Strongly typed inductive concept learning. In D. Page, editor, Proceedings of the Eighth International Conference on Inductive Logic Programming, volume 1446, pages 185-194. Springer-Verlag, 1998.

11. G Gallo, G Longo, S Pallottino, and Sang Nguyen. Directed hypergraphs and applications. Discrete Applied Mathematics, 42:177-201, 1993.

12. M. T. Goodrich and R. Tamassia. Algorithm Design. Wiley, 2002.

13. Masaki Ito and Hayato Ohwada. Efficient database access for implementing a scalable ILP engine. In Work-In-Progress Report of the Eleventh International Conference on Inductive Logic Programming, 2001.

14. N. Jacobs and H. Blockeel. From shell logs to shell scripts. In Proceedings of ILP2001 - Eleventh International Workshop on Inductive Logic Programming, volume 2157 of Lecture Notes in Artificial Intelligence, pages 80-90, 2001.

15. K. Morik and P. Brockhausen. A multistrategy approach to relational discovery in databases. Machine Learning, 27(3):287-312, 1997.

16. S. Muggleton. Inverse entailment and Progol. New Generation Computing, Special issue on Inductive Logic Programming, 13(3-4):245-286, 1995.

17. S. Muggleton, R.D. King, and M.J.E. Sternberg. Protein secondary structure prediction using logic-based machine learning. Protein Engineering, 7:647-657, 1992.

18. J. Ramon, T. Francis, and H. Blockeel. Learning a Tsume-Go heuristic with Tilde. In Proceedings of CG2000, the Second international Conference on Computers and Games, volume 2063 of Lecture Notes in Computer Science, pages 151-169. Springer-Verlag, 2000.

19. J. Seitzer, J. P. Buckley, and Y. Pan. INDED: A distributed knowledge-based learning system. IEEE Intelligent Systems, 15(5):38-46, 2000. 